\title{
ON THE PROBABILITY DISTRIBUTION OF FREAK WAVES IN FINITE WATER DEPTH
}

\author{
Kyugmo Ahn ${ }^{1}$, S. Kim² and S. Cheon ${ }^{3}$
}

\begin{abstract}
This paper presents the occurrence probability of freak waves based on the analysis of extensive wave data collected during ARSLOE project. It is suggested to use the probability distribution of extreme waves heights as a possible means of defining the freak wave criteria instead of conventional definition which is the wave height greater than the twice of the significant wave height. Analysis of wave data provided such finding as 1) threshold tolerance of $0.2 \mathrm{~m}$ is recommended for the discrimination of the false wave height due to noise 2) no supportive evidence on the linear relationship between the occurrence probability of freak waves and the kurtosis of surface elevation 3) nonlinear wave-wave interactions are not the primary cause of the generation of freak waves 4) the occurrence of freak waves does not depend on the wave period 5) probability density function of extreme waves can be used to predict the occurrence probability of freak waves. Three different distribution functions of extreme wave height by Rayleigh, Ahn, and Mori were compared for the analysis of freak waves.
\end{abstract}

Keywords: freak wave; occurrence probability; extreme wave; significant wave height

\section{INTRODUCTION}

The construction of the probability density function of wave heights is one of the most important tasks in the study of wind-generated waves. In addition, the estimation of the extreme wave heights is necessary for the probabilistic forecasting and warning of hazards from freak waves. It is well known that freak waves inflict severe damages upon offshore structures, ships, and marine equipments. This phenomenon is rare but critically meaningful. Hence, acquiring an adequate knowledge of the maximum expected wave heights, especially including unusual cases such as freak waves, is required for the prediction of wave forces and structural responses related to the design of structures in the ocean.

A freak wave is an interesting but difficult topic to figure out. Many scientist and engineers have shown great interest to the possible mechanism of freak waves and have published the results of their research work. Despite many efforts to understand plausible causes, the mechanisms as well as statistical characteristic of freak waves have not yet been clearly resolved.

It is imperative to clarify the definition and concept of a freak wave. A freak wave (also known as a rogue wave) is "commonly" defined as a single wave height which is more than twice the significant wave height. However, there remains the problem of not defining accurate characteristics of a freak wave. Recently, Liu et al. (2009) raised a question on "what is a freak wave?"; they claim that as neither freak nor rogue waves have been clearly defined, it is only vaguely implying some kind of unexpected, larger than usual waves in the field. They coined a portmanteau word "freaque" waves derived from combining the words "freak" and "rogue" waves. The word has two additional implications: first, it is a kind of steep elevated abnormal wave, and second, freque waves are not analogous to the extreme waves, because a freaque wave may be a local extreme, but most extreme waves are not al freague waves.

Moreover, a loosely used term "the significant wave height" makes it harder to define a freak wave. This problem has been largely ignored. First, using the Rayleigh distribution, its value is widely known to be equal to four times the standard deviation of the surface elevation $\sigma$,

$$
H_{s}=(3 \sqrt{2 \pi} \operatorname{erfc}(\sqrt{\ln 3}+2 \sqrt{2 \ln 3})) \sigma \simeq 4 \sigma
$$

where $\operatorname{erfc}(x)$ is the error function. This relationship is valid only when the distribution of surface elevation follows the Gaussian random process. Second, the significant wave height is defined as the average of the highest $1 / 3$ of the waves, $H_{1 / 3}$, which should be estimated based on the wave-by-wave analysis in time domain. In reality, however, $H_{1 / 3}$ is not always identical to $H_{\mathrm{s}} \approx 4.0 \sigma$, when waves are deviated from the assumption of Gaussian random processes and Rayleigh distributions. Therefore, when $H_{1 / 3}$ and $H_{\mathrm{s}}$ are not identical, $H_{1 / 3}$ which does not depend on the assumption of Gaussian random processes, rather than $H_{\mathrm{s}}$, should be used in the analysis. With these considerations in mind, a freak wave denoted by $H_{\text {freak }}$ is consequently determined from

$$
H_{\text {freak }} \geq 2 H_{1 / 3} \text {. }
$$

\footnotetext{
${ }^{1}$ Handong Global University, School of Spatial Environment System Engineering, Pohang, Kyeongbuk-Do, 791-708 Korea,kmahn@handong.edu

2 Institute of Construction \& Environmental Research, Pohang, Kyeongbuk-Do, 791-708, Korea

${ }^{3}$ Department of Civil and Environmental Engineering, Seoul National University., 1 Gwanak-ro, Gwanak-gu, Seoul, 151-774, Korea
} 
Various researches on the freak waves which have been conducted during the past decades can be largely divided into two major categories - one is the deterministic approaches and the other is the statistical ones. Researches adopting the deterministic approaches on the freak waves are as follows: Henderson et al. (1999) investigated a fully nonlinear numerical solution of the nonlinear Schrödinger equation and draw a comparison with an exact solution. Kharif and Pelinovsky (2003)'s studies give convincing answers to many questions of the freak wave events in light of the deterministic approaches. On the other hand, statistical approaches to predict the occurrence probability of freak waves were summarized by Mori and Janssen (2006). Janssen provided an overview of the relation between nonlinear four-wave interactions and freak waves in the context of the stochastic approach (Janssen 2003). Mori's overall result need to be described in detail. Yasuda, and Mori (1997) emphasized that the occurrence probability of the freak waves could be argued generally under the Rayleigh extreme distribution, although its validity varies from area to area. They judged that the classic method, in which the largest maximum individual wave height is given as the most probable maximum wave height in the severest sea state with the largest of $H_{1 / 3}$, is underestimated. Mori et al. (2002) analyzed that the crest and trough amplitude distributions of the observed ocean waves including freak waves are different from the Rayleigh distribution, although the wave height distribution tends to agree with the Rayleigh distribution. To be specific, the probability from the Rayleigh distribution is said to be underestimated for crest amplitude but is over-estimated for trough amplitudes. Thereby, they developed the EdgeworthRayleigh distribution instead of the Rayleigh distribution for the estimation of occurrence of freak waves. They presented the wave height distribution as a function of the fourth cumulant (or kurtosis) $\mu_{4}$ and the third cumulant (or skewness) $\mu_{3}$. By using the joint probability density function of the sea surface elevation and its Hilbert transform, they developed the probability density function of wave height semiempirically for narrow banded weakly nonlinear waves as follows:

$$
g\left(H^{\prime}\right) d H^{\prime}=\frac{H^{\prime}}{4} \exp \left(-\frac{H^{\prime 2}}{8}\right)\left[1+\sum_{i, j} \beta_{i, j} B_{i, j}\left(H^{\prime}\right)\right] d H^{\prime}
$$

where $H^{\prime}$ is the wave height $H$ normalized by the room-mean-square of the surface elevation $\eta_{\mathrm{rms}} . \beta_{\mathrm{i}, \mathrm{j}}$ are coefficients containing the skewness and kurtosis of the water surface elevation and $B_{\mathrm{i}, \mathrm{j}}$ are polynomials in terms of $H^{\prime}$. Mori (2004) suggested a formula for the extreme wave height, which is derived from the above equation using a non-Gaussian theory for a unidirectional wave train with narrow banded spectra to predict the occurrence probability of freak waves. He insisted on the enhancement of the occurrence probability of freak waves due to rise of the fourth order moment of surface elevation, kurtosis. He also argued that the nonlinear effects on the occurrence probability of freak waves linearly depends on kurtosis for a small number of waves $\mathrm{N}=250$.

Stansell (2005) investigated statistical models for the relative occurrence of the most extreme wave, crest and trough heights measured during severe storm conditions in deep water. He used the fitted Generalized Pareto distribution (GPD) and examines the differences between the predictions of the Rayleigh probability density function and those of the GPD. According to this paper, the fitted GPDs showed that the Rayleigh distribution is inadequate for modeling the probability of occurrence of extremes of the dimensionless crest or trough heights.

\section{ANALYSIS OF WAVE DATA INCLUDING FREAK WAVES}

In this study, wave records containing about 900,000 individual waves were analyzed to find freak waves. This data sample was measured during the period of an extratropical cyclone. The data were obtained from the Atlantic Ocean Remote Sensing Land Ocean Experiment (ARSLOE) project by the Coastal Engineering Research Center, U.S. Army Corps of Engineers, at its Field Research Facility, located at Duck, North Carolina. The sampling frequency of wave data is $4 \mathrm{~Hz}$ which corresponds to sampling interval of $0.25 \mathrm{sec}$. Wave data were collected continuously and each wave records has a duration of 20 minutes. Fig. 1 shows the water depth and distances from the shoreline of 8 wave gages deployed during ARSLOE project. 


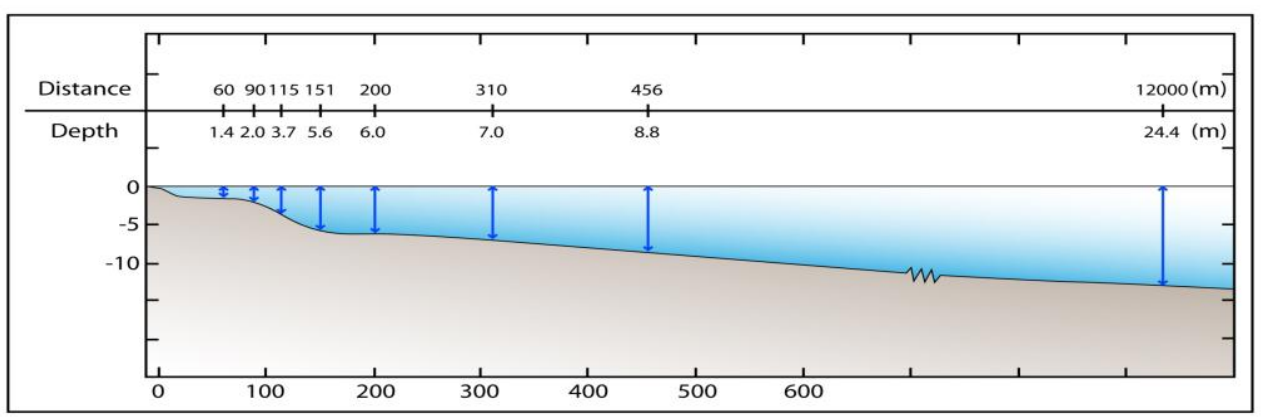

Fig. 1 Water depths and distances from shoreline of the 8 wave gages deployed during ARSLOE project.

\section{Zero-Up-Crossing Analysis}

When we apply zero-up-crossing analysis to the observed raw wave data in order to identify individual wave height, it is necessary to eliminate false wave height due to noise. For example, wave heights of $0.1 \mathrm{~m}$ with short wave period might be considered to be noise rather than to be individual wave height. These noise could be eliminated through low-pass filtering. However, there is no universally accepted threshold values that can be set to the limit of noise. Low pass filtering using smoothing technique is used to eliminate the noise with threshold values of $0.1 \mathrm{~m}$ and $0.2 \mathrm{~m}$, respectively. Fig. 2 shows the time series of wave data where the green line illustrates raw data and the red and blue lines represent filtered with threshold value of $0.1 \mathrm{~m}$ and $0.2 \mathrm{~m}$, respectively.

Judging from Fig. 2, it is suggested that the suitable threshold value is $0.2 \mathrm{~m}$ by considering more reasonable and smooth wave profile. However, this study shall analyze the ARSLOE wave data for both $0.1 \mathrm{~m}$ and $0.2 \mathrm{~m}$ cases in order to illustrate the importance of the threshold values in freak wave analysis.
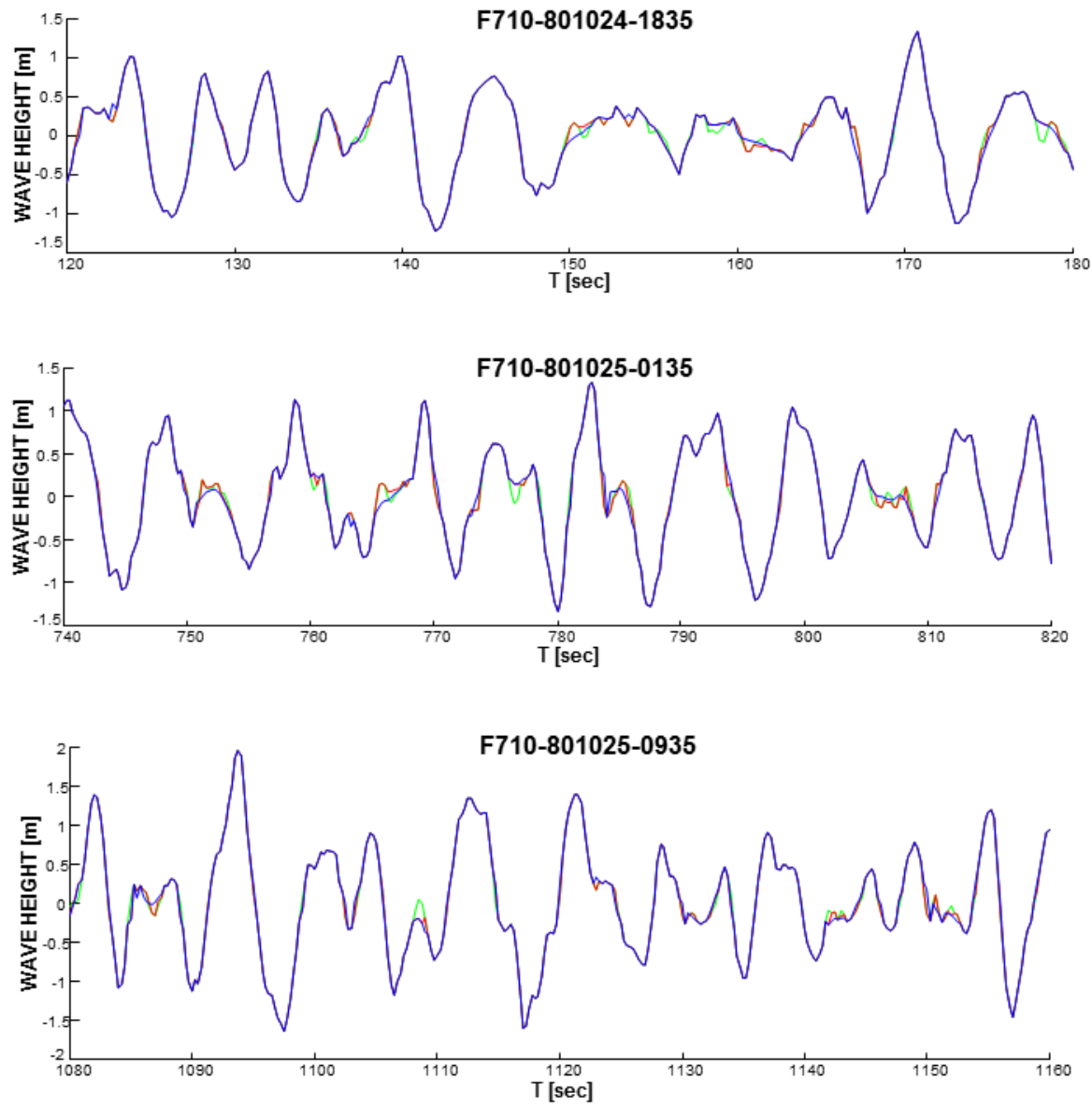

Fig. 2 A portion of wave record : Raw data (green) and wave profile with threshold tolerance of $0.1 \mathrm{~m}$ (red) and $0.2 \mathrm{~m}$ (blue). 
For the Case of Threshold Tolerance of $0.1 \mathrm{~m}$

The ARSLOE wave data had been collected at 8 different locations with wave gages aligned perpendicular to the shoreline during Oct. 24 to 26, 1980. Wave data analyzed in this study were obtained at 8 different water depth from most shallow water depth of about $2.1 \mathrm{~m}$ to the deepest of water depth of about $25 \mathrm{~m}$ which correspond to the distance from $60 \mathrm{~m}$ up to $12 \mathrm{~km}$ away from the shoreline.

When the ARSLOE wave data are filtered with the threshold limits of $0.1 \mathrm{~m}$, there are four freak waves found in the data; (a) Oct. 24th, 16:55, (b) Oct. 24th, 18:35, (c) Oct. 25th, 01:35, and (d) Oct. 25th, 09:35. Four freak waves were found only at station F710 which is the deepest water depth of about $24 \mathrm{~m}$. No freak waves were found in wave data collected at other 7 wave gages. Summary of the four freak waves are outlined in Table 1. Time series plot of the surface elevations are shown in Fig. 3.

It is interesting to see the ratio $H_{\mathrm{rms}} / H_{1 / 3}$ of two cases, (b) Oct. 24th, 18:35, and (c) Oct. 25th, 01:35 in Table 1. The ratio of 2.002 and 2.007, respectively, barely meet the criteria of freak wave.

\begin{tabular}{|c|cccc|}
\hline $\begin{array}{l}\text { Table 1. A summary of parameters for the perceived four freak wave cases with the threshold } \\
\text { tolerance of } \mathbf{0 . 1} \mathbf{~ m} \text {. }\end{array}$ & (a) $24^{\text {th }}, 16: 55$ & (b) $24^{\text {th }}, 18: 35$ & (c) $25^{\text {th }}, 01: 35$ & (d) $25^{\text {th }}, 09: 35$ \\
\hline Date & 212 & 213 & 196 & 177 \\
\hline No. of Waves & 2.26 & 2.002 & 2.007 & 2.28 \\
$H_{\max } / H_{1 / 3}$ & $5.71 \mathrm{~m}$ & $5.32 \mathrm{~m}$ & $5.72 \mathrm{~m}$ & $9.09 \mathrm{~m}$ \\
$H_{\max }$ & $2.52 \mathrm{~m}$ & $2.65 \mathrm{~m}$ & $2.85 \mathrm{~m}$ & $3.98 \mathrm{~m}$ \\
$H_{1 / 3}$ & $1.65 \mathrm{~m}$ & $1.72 \mathrm{~m}$ & $1.81 \mathrm{~m}$ & $2.48 \mathrm{~m}$ \\
$H_{\operatorname{mean}}$ & $1.83 \mathrm{~m}$ & $1.91 \mathrm{~m}$ & $2.03 \mathrm{~m}$ & $2.82 \mathrm{~m}$ \\
$H_{\text {rms }}$ & $6.75 \mathrm{sec}$ & $7 \mathrm{sec}$ & $7.75 \mathrm{sec}$ & $9.5 \mathrm{sec}$ \\
Wave period of $H_{\max }$ & $5.64 \mathrm{sec}$ & $5.59 \mathrm{sec}$ & $6.08 \mathrm{sec}$ & $6.69 \mathrm{sec}$ \\
Average wave period & -0.0149 & 0.0112 & -0.0041 & 0.0708 \\
\hline Skewness & 3.4145 & 3.2805 & 3.2330 & 3.5032 \\
\hline Kurtosis & & & & \\
\hline
\end{tabular}

The scatter plots for the relationship between wave heights and periods obtained from the wave records containing for four freak waves are shown in Fig. 4. As is shown in the figure, the pink points are freak waves which do not match with the longest or the shortest wave period. Therefore, we may conclude that the occurrence of freak wave does not depend on the wave period.

\section{For the Case of Threshold Tolerance of $0.2 \mathrm{~m}$}

When the ARSLOE wave data are filtered with the threshold limit of $0.2 \mathrm{~m}$, there are only two freak waves found in the data; (a) Oct. 24th, 16:55 and (d) Oct. 25th, 09:35. When we filtered out small waves which are considered to be noise, the reduced total number of waves in the wave record result in higher significant wave height. As a result, the ratio $H_{\mathrm{rms}} / H_{1 / 3}$ should be reduced. In the following Table 2, the numbers in parenthesis are from the previous Table 1 for easy comparison of changes.

\begin{tabular}{|c|c|c|}
\hline Date & (a) $24^{\text {th }}, 16: 55$ & (d) $25^{\text {th }}, 09: 35$ \\
\hline No. of Waves & $196(212)$ & $162(177)$ \\
\hline$H_{\max } / H_{1 / 3}$ & $2.21(2.26)$ & $2.21(2.28)$ \\
\hline$H_{\max }$ & $5.71 \mathrm{~m}$ & $9.09 \mathrm{~m}$ \\
\hline$H_{1 / 3}$ & $2.57 \mathrm{~m}(2.52 \mathrm{~m})$ & $4.10 \mathrm{~m}(3.98 \mathrm{~m})$ \\
\hline$H_{\text {mean }}$ & $1.75 \mathrm{~m}(1.65 \mathrm{~m})$ & $2.63 \mathrm{~m}(2.48 \mathrm{~m})$ \\
\hline$H_{\text {rms }}$ & $1.90 \mathrm{~m}(1.83 \mathrm{~m})$ & $2.95 \mathrm{~m}(2.82 \mathrm{~m})$ \\
\hline Wave period of $H_{\max }$ & $6.75 \mathrm{sec}$ & $9.75 \mathrm{sec}$ \\
\hline Average wave period & $6.11 \mathrm{sec}(5.64 \mathrm{sec})$ & $7.31 \mathrm{sec}(6.69 \mathrm{sec})$ \\
\hline
\end{tabular}



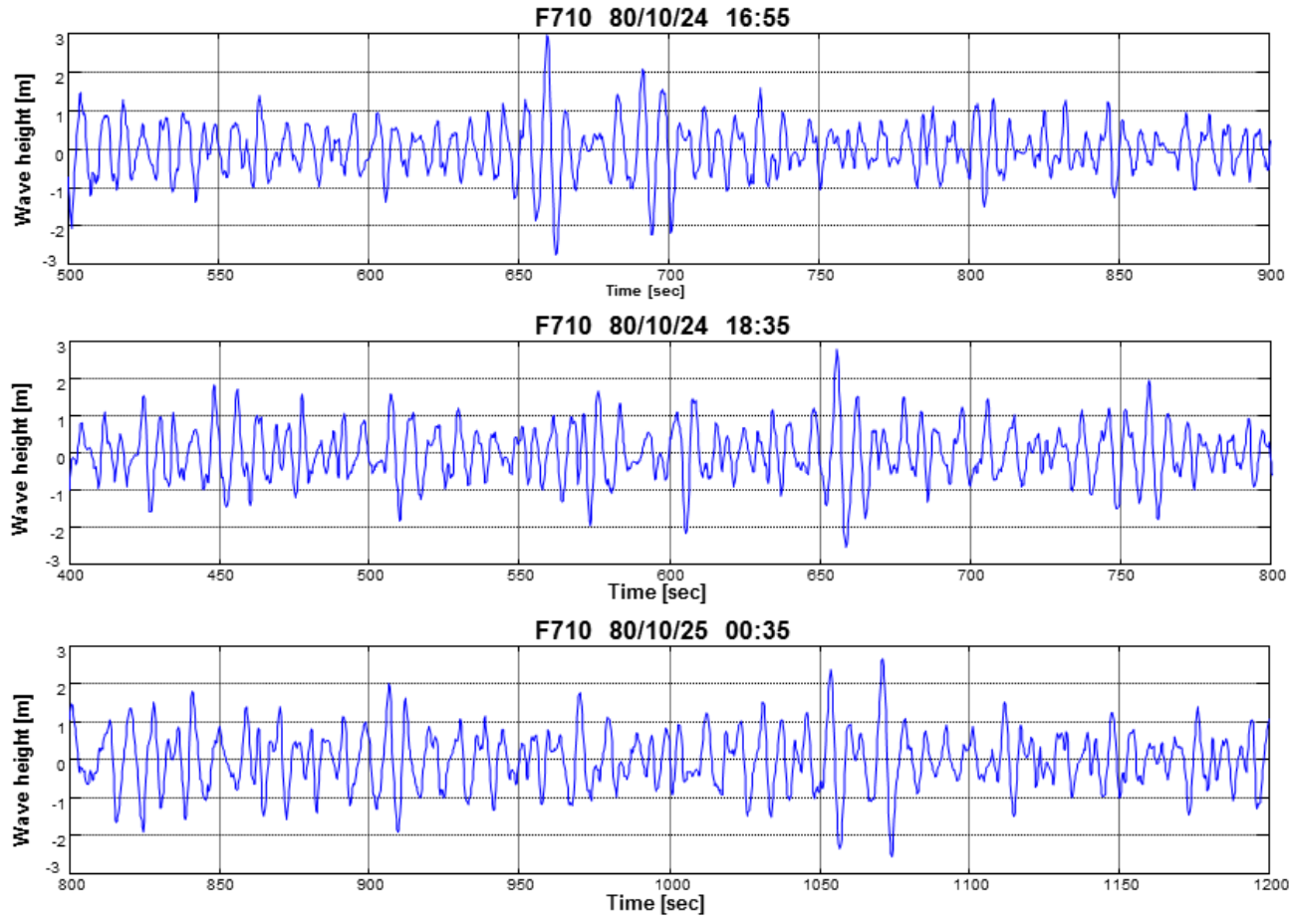

F710 $80 / 10 / 25 \quad 09: 35$

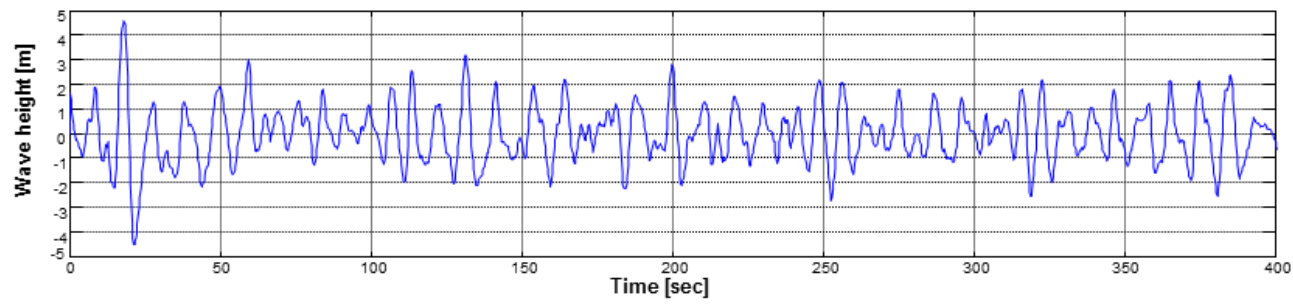

Fig. 3. Time-series of surface elevation including four freak waves (with filtering threshold of $0.1 \mathrm{~m}$ )
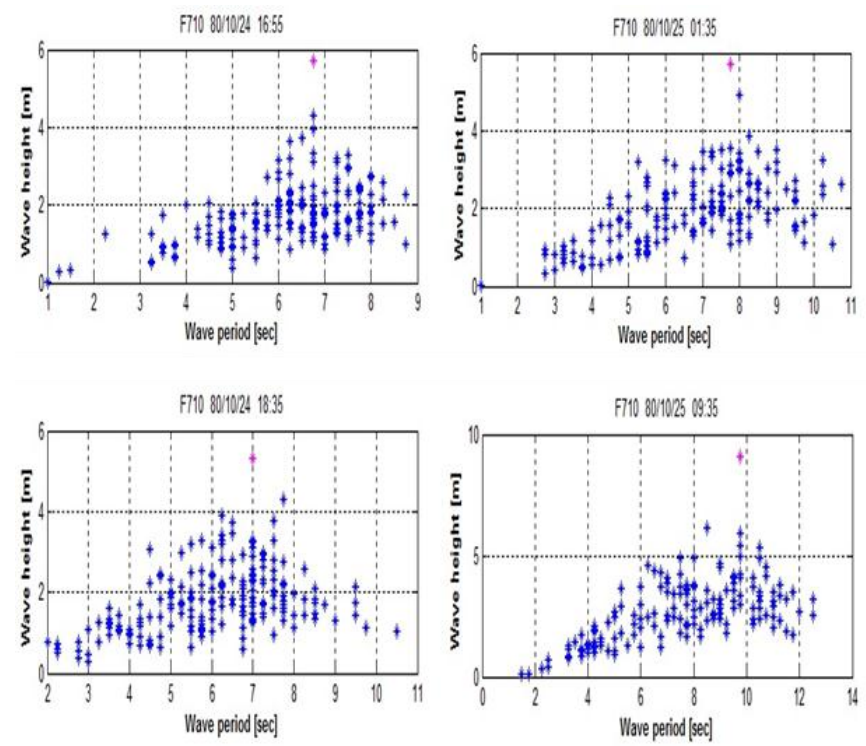

Fig. 4. Scatter plot between the wave period and wave height obtained from wave-by-wave analysis of the wave record which contains freak waves (pink points denote a freak wave) 
Probability Distribution of Extreme Wave Heights

For the statistical analysis of freak waves, three different probability density function of the extreme (largest) wave height are compared. The probability density function of extreme waves can be derived from the probability density function of wave height using order statistics. (Ochi ****) Three probability density functions of extreme wave heights are by Rayleigh, Ahn, and Mori.

\section{Probability Density function of Extreme Wave Heights based on the Rayleigh distribution}

The probability density function of extreme wave heights based on the Rayleigh probability density function is as following:

$$
g\left(H_{\max }\right)=n\left(\frac{2 H_{n}}{m_{2}} \exp ^{-\frac{H_{\max }^{2}}{m_{2}}}\right)\left(1-\exp ^{-\frac{H_{\max }^{2}}{m_{2}}}\right)^{n-1}
$$

where $m_{2}$ is the second moment of the wave height, $n$ is the number of waves.

\section{Probability Density function of Extreme Wave Heights by Ahn}

The probability density function of extreme wave heights in $n$ observations is derived by Ahn (2002) based on the maximum entropy method as follows;

$$
g\left(H_{\max }\right)=n\left(\frac{\sqrt{\pi}}{2 \sqrt{\lambda_{2}}}\right)^{n-1} A^{n} e^{-\lambda_{2}\left(H_{\max }+\lambda_{1} / 2 \lambda_{2}\right)^{2}}\left\{\operatorname{erf}\left(\sqrt{\lambda_{2}}\left(H_{n}+\frac{\lambda_{2}}{2 \sqrt{\lambda_{2}}}\right)\right)-\operatorname{erf}\left(\frac{\lambda_{1}}{2 \sqrt{\lambda_{2}}}\right)\right\}^{n-1}
$$

Where $A=\exp \left(-\lambda_{0}-\lambda_{1}^{2} / 4 \lambda_{2}\right), \lambda_{0}, \lambda_{1}$, and $\lambda_{2}$ are parameters which can be calculated from the first and second moment of wave heights.

\section{Probability Density function of Extreme Wave Heights by Mori}

Mori (2004) developed the distribution of the maximum wave height of the nonlinear wave field in a unidirectional wave train as follows;

$$
\begin{aligned}
g\left(H_{\max }\right)= & n \frac{H_{\max }}{4} \exp \left\{-\frac{H_{\max }^{2}}{8}-n_{0} \exp \left(-\frac{H_{\max }^{2}}{8}\right)\left[1+\sum_{i, j} \beta_{i, j} E_{i, j}\left(H_{\max }\right)\right]\right\} \\
& \times\left[1+\sum_{i, j} \beta_{i, j} E_{i, j}\left(H_{\max }\right)\right] d H_{\max }
\end{aligned}
$$

where $\beta_{\mathrm{i}, \mathrm{j}}$ are coefficients containing the skewness $\mu_{3}$ and kurtosis $\mu_{4}$ of the water surface elevation $\eta(t)$.

\section{The Occurrence Probability of Freak Waves}

As analyzed in the previous section, freak waves were found only in the wave gage F710 which is located in the deepest water depth. No freak waves were found in the intermediate as well as shallow water depth waves. It is well known that as waves propagate from deep to shallow water depth, nonlinear characteristics of waves become more pronounced and become non-Gaussian random waves in shallow water depth.

It is interesting to note that freak waves are more likely to be generated in deep water waves compared to shallower water depth. This also indicates that freak waves are more likely to be generated when nonlinearity and non-Gaussian characteristic of waves are weak. These are rather a surprise and even contrary to the generally accepted findings such that the main cause of freak wave generation is due to nonlinear wave-wave interactions (Oalgnon and Athanassouls, 2000) This is also contrary to the Mori's assertion that probability of occurrence of freak waves increases as the kurtosis of surface elevation increases.

Fig. 5 shows the comparison between histogram of wave height and the probability density function of wave height by Ahn, Rayleigh, and Mori. The probability density functions of extreme wave height by Ahn, Rayleigh, and Mori are also shown in the figure. 

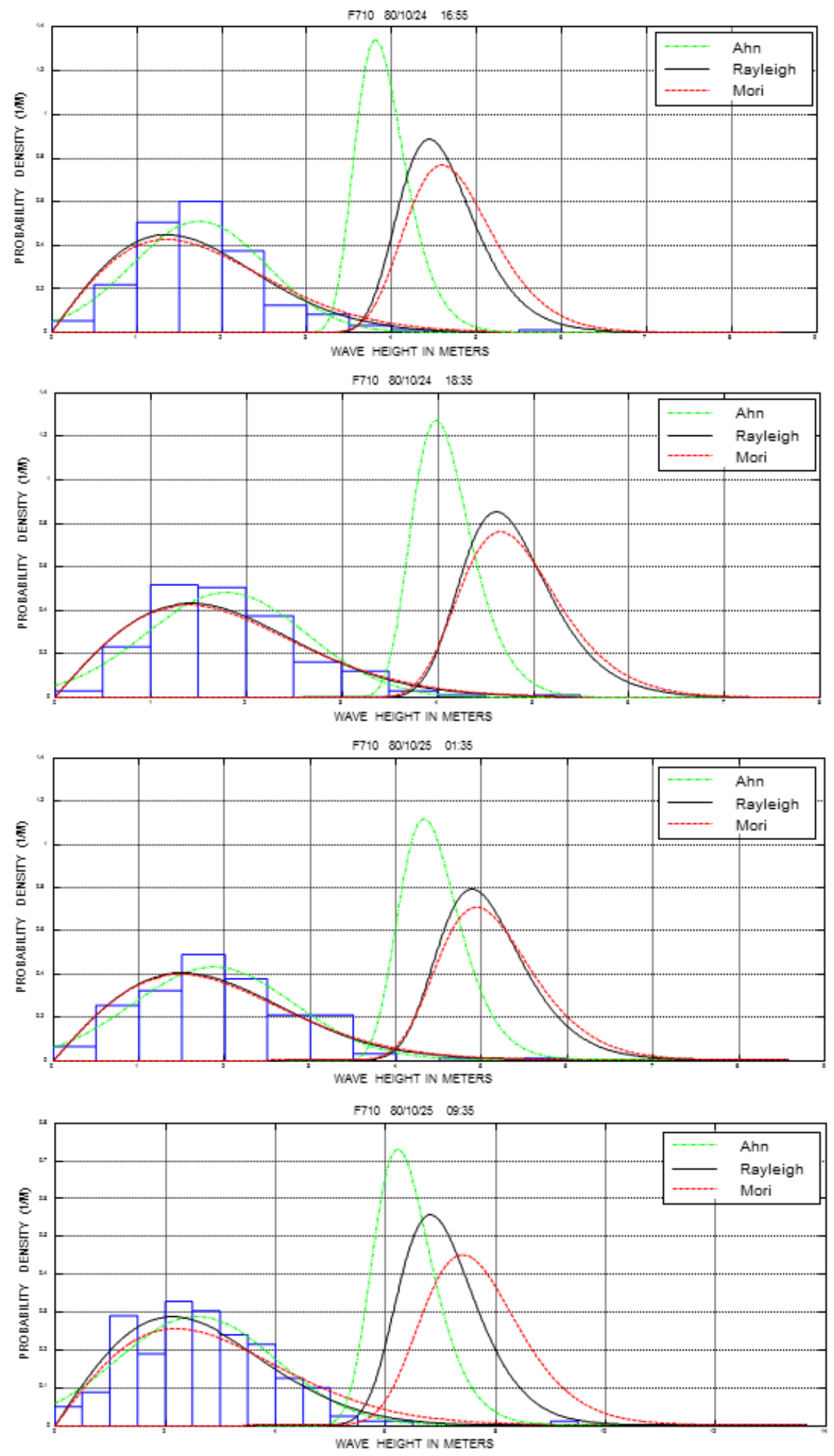

Fig. 5. Comparison between histogram of wave height and the probability density function of wave height by Ahn, Rayleigh, and Mori. The probability density functions of extreme wave height by Ahn, Rayleigh, and Mori are also shown in the figure.

In order to understand the occurrence probability of freak waves, the extreme wave heights of total 134 wave data records at wave gage F710 during the period from October 24 00:15 to October 26 00:35, 1980 were analyzed. The occurrence probability of extreme waves using 3 different distribution functions based on Raylerigh, Ahn, and Mori were computed. 
For the Case of Threshold Tolerance of $0.1 \mathrm{~m}$

The occurrence probability of four freak wave cases are represented as percentages in Table 3 . Included also in the table is the occurrence probability of more than $2 H_{1 / 3}$, which is exceedence probability of $2 H_{1 / 3}$. It is found that the probability of occurrence of freak waves are much less than those of exceedence of $2 \mathrm{H}_{1 / 3}$.

\begin{tabular}{|c|c|c|c|}
\hline \multicolumn{4}{|c|}{ Occurrence probability more than $H_{\max }$} \\
\hline Date & Rayleigh & Ahn & Mori \\
\hline (a) $24^{\text {th }}, 16: 55$ & $1.375 \%$ & $0.032 \%$ & $6.432 \%$ \\
\hline (b) $24^{\text {th }}, 18: 35$ & $8.734 \%$ & $0.476 \%$ & $19.890 \%$ \\
\hline (c) $25^{\text {th }}, 01: 35$ & $6.895 \%$ & $0.636 \%$ & $17.556 \%$ \\
\hline (d) $25^{\text {th }}, 09: 35$ & $0.547 \%$ & $0.071 \%$ & $8.466 \%$ \\
\hline \multicolumn{4}{|c|}{ Occurrence probability more than $2 H_{1 / 3}$} \\
\hline Date & Rayleigh & Ahn & Mori \\
\hline (a) $24^{\text {th }}, 16: 55$ & $10.765 \%$ & $0.946 \%$ & $29.657 \%$ \\
\hline (b) $24^{\text {th }}, 18: 35$ & $8.907 \%$ & $0.492 \%$ & $20.190 \%$ \\
\hline (c) $25^{\text {th }}, 01: 35$ & $7.272 \%$ & $0.691 \%$ & $18.280 \%$ \\
\hline (d) $25^{\text {th }}, 09: 35$ & $5.828 \%$ & $1.685 \%$ & $36.571 \%$ \\
\hline
\end{tabular}

The occurrence probability of extreme waves, $H_{\max }$, were calculated for total 134 wave data sets from October 24 00:15 to October 26 00:35 at twenty-minute intervals. Based on each formula, they were sorted into ascending values. For the case of threshold tolerance of $0.1 \mathrm{~m}$, the only result obtained by the probability distribution of extreme wave height based on Rayleigh distribution put four freak waves in consecutive order, as illustrated in Table 4.

\begin{tabular}{|c|c|c|c|c|}
\hline \multicolumn{5}{|c|}{ Occurrence probability more than $H_{\max }$} \\
\hline Rank & Date & Rayleigh & Ahn & Mori \\
\hline 1 & (d) $25^{\text {th }}, 09: 35$ & $0.547 \%$ & $0.071 \%$ & $8.466 \%$ \\
\hline 2 & (a) $24^{\text {th }}, 16: 55$ & $1.375 \%$ & $0.032 \%$ & $6.432 \%$ \\
\hline 3 & (c) $25^{\text {th }}, 01: 35$ & $6.895 \%$ & $0.636 \%$ & $17.556 \%$ \\
\hline 4 & (b) $24^{\text {th }}, 18: 35$ & $8.734 \%$ & $0.476 \%$ & $19.890 \%$ \\
\hline 5 & $25^{\text {th }}, 15: 35$ & $13.556 \%$ & $1.548 \%$ & $20.661 \%$ \\
\hline 6 & $25^{\text {th }}, 00: 15$ & $13.983 \%$ & $0.788 \%$ & $26.767 \%$ \\
\hline 7 & $25^{\text {th }}, 00: 35$ & $14.925 \%$ & $0.238 \%$ & $10.507 \%$ \\
\hline 8 & $24^{\text {th }}, 06: 15$ & $17.302 \%$ & $0.835 \%$ & $37.481 \%$ \\
\hline 9 & $25^{\text {th }}, 07: 15$ & $18.034 \%$ & $3.535 \%$ & $38.327 \%$ \\
\hline 10 & $25^{\text {th }}, 11: 55$ & $18.622 \%$ & $4.245 \%$ & $31.783 \%$ \\
\hline
\end{tabular}


For the Case of Threshold Tolerance of $0.2 \mathrm{~m}$

Through the same process shown in the previous section but with different threshold tolerance of $0.2 \mathrm{~m}$, the probability distributions of extreme wave height of three different distribution functions are provided in Table 5. The first part of the table is sorted ascending order by Rayleigh distribution and the second one is by Ahn's and finally the third one is by Mori's. All 3 different distributions correctly discriminate the freak waves with the probability of occurrence of less than $3 \%, 0.1 \%$ and $8 \%$. The implication of this result is such that we may utilize the probability distribution function of extreme waves to predict the occurrence probability of freak waves at specific locations in the ocean.

\begin{tabular}{|c|c|c|c|c|}
\hline Rank & Date & Rayleigh & Ahn & Mori \\
\hline 1 & (d) $25^{\text {th }}, 09: 35$ & $1.263 \%$ & $0.064 \%$ & $7.815 \%$ \\
\hline 2 & (a) $24^{\text {th }}, 16: 55$ & $2.510 \%$ & $0.005 \%$ & $6.053 \%$ \\
\hline 3 & (c) $25^{\text {th }}, 01: 35$ & $11.161 \%$ & $0.380 \%$ & $16.523 \%$ \\
\hline 4 & (b) $24^{\text {th }}, 18: 35$ & $12.602 \%$ & $0.244 \%$ & $19.036 \%$ \\
\hline 5 & $25^{\text {th }}, 00: 15$ & $17.345 \%$ & $0.771 \%$ & $26.163 \%$ \\
\hline Rank & Date & Rayleigh & $A h n$ & Mori \\
\hline 1 & (a) $24^{\text {th }}, 16: 55$ & $2.510 \%$ & $0.005 \%$ & $6.053 \%$ \\
\hline 2 & (d) $25^{\text {th }}, 09: 35$ & $1.263 \%$ & $0.064 \%$ & $7.815 \%$ \\
\hline 3 & $25^{\text {th }}, 00: 35$ & $21.905 \%$ & $0.087 \%$ & $9.964 \%$ \\
\hline 4 & (b) $24^{\text {th }}, 18: 35$ & $12.602 \%$ & $0.244 \%$ & $19.036 \%$ \\
\hline 5 & (c) $25^{\text {th }}, 01: 35$ & $11.161 \%$ & $0.380 \%$ & $16.523 \%$ \\
\hline Rank & Date & Rayleigh & Ahn & Mori \\
\hline 1 & (a) $24^{\text {th }}, 16: 55$ & $2.510 \%$ & $0.005 \%$ & $6.053 \%$ \\
\hline 2 & (d) $25^{\text {th }}, 09: 35$ & $1.263 \%$ & $0.064 \%$ & $7.815 \%$ \\
\hline 3 & $25^{\text {th }}, 00: 35$ & $21.905 \%$ & $0.087 \%$ & $9.964 \%$ \\
\hline 4 & $24^{\text {th }}, 08: 15$ & $49.288 \%$ & $0.626 \%$ & $15.139 \%$ \\
\hline 5 & $24^{\text {th }}, 02: 35$ & $24.218 \%$ & $0.607 \%$ & $15.991 \%$ \\
\hline
\end{tabular}

\section{DISCUSSION}

As is presented in the previous sections, it is thus tempting to conclude that the threshold tolerance of $0.2 \mathrm{~m}$ is more appropriate to determine the occurrence probability of a freak wave in the ARSLOE wave data analyzed in this study. The conventional definition of freak wave, $H_{\text {freak }} \geq 2 H_{1 / 3}$, strongly depends on the significant wave height which is also dependent on the number of waves in the records. Thereby, noise signals result in an increase of number of waves due to false wave heights which tend to over-predict the significant waves height.

ARSLOE wave data analysis shows that the exceedence probability of $2 H_{1 / 3}$ is higher than that of freak waves. Therefore, it is more reasonable to define freak waves based on the probability occurrence of extreme wave heights.

As mentioned in Introduction, Mori insisted on the enhancement of the occurrence probability of freak waves due to the rise of the fourth order moment of surface elevation, kurtosis. However, his conclusion did not sustain scrutiny. For a critical examination of the relationship between occurrence probability and kurtosis, no supportive evidence is observable. The Table 6 illustrates top ten kurtosis values in ascending order and the corresponding ratio $H_{\max } / H_{1 / 3}$ as an indicator of a freak wave obtained from wave gage F710. As can be seen in the table, high kurtosis does not coincide with freak waves and no apparent linearity can be found between kurtosis and the ratio $H_{\max } / H_{1 / 3}$. During the same period of storm, freak waves were found only in deep water waves. This also does not support the Mori's assertion.

The probability distribution of extreme wave heights suggested by Rayleigh, Ahn, and Mori can be used to predict the probability occurrence of freak waves. However, there is no definite measures available in comparing the accuracy in predicting the freak waves among three distribution function. For doing this we need more wave data needed to compare the performance of the predicting capability. Especially, long term wave data which satisfy the stationarity of time series is necessary. 


\begin{tabular}{|c|c|c|c|}
\hline \multicolumn{4}{|c|}{$\begin{array}{l}\text { Table 6. Top ten kurtosis values in descending order with the ratio } H_{\max } / H_{1 / 3} \text { (with } \\
\text { threshold tolerance of } 0.2 \mathrm{~m} \text { ) obtained from } 134 \text { data set at F710. }\end{array}$} \\
\hline Rank & kurtosis & Date & ratio \\
\hline 1 & 3.503 & (d) $25^{\text {th }}, 09: 35$ & 2.215 \\
\hline 2 & 3.496 & $24^{\text {th }}, 19: 35$ & 1.735 \\
\hline 3 & 3.451 & $24^{\text {th }}, 06: 15$ & 1.914 \\
\hline 4 & 3.414 & (a) $24^{\text {th }}, 16: 55$ & 2.215 \\
\hline 5 & 3.396 & $25^{\text {th }}, 15: 15$ & 1.684 \\
\hline 6 & 3.349 & $25^{\text {th }}, 00: 15$ & 1.916 \\
\hline 7 & 3.340 & $25^{\text {th }}, 19: 15$ & 1.659 \\
\hline 8 & 3.337 & $25^{\text {th }}, 08: 55$ & 1.812 \\
\hline 9 & 3.336 & $24^{\text {th }}, 09: 15$ & 1.666 \\
\hline 10 & 3.314 & $25^{\text {th }}, 20: 15$ & 1.743 \\
\hline
\end{tabular}

\section{SUMMAY AND CONCLUSIONS.}

In this study, ARSLOE wave data records which contain 900,000 individual waves were analyzed to find freak waves. The wave data collected from 8 wave gages deployed water depth from $2.1 \mathrm{~m}$ to $24 \mathrm{~m}$ during storm provided valuable information on the nature of freak waves. Only 2 freak waves found in the water depth of $24 \mathrm{~m}$ which is the deepest water depth.

It is found that the threshold tolerance of $0.2 \mathrm{~m}$ is appropriate to determine a freak wave in the ARSLOE wave data analyzed in this study. It is also recommended to define freak waves based on the probability distribution of extreme waves instead of the conventional definition of freak wave, $H_{\text {freak }} \geq 2 H_{1 / 3}$.

According to the analysis of ARSLOE wave data, there is no supportive evidence available on the linear relationship between the occurrence probability of freak waves and the fourth order moment of surface elevation, kurtosis. It is also found that nonlinear wave-wave interactions might not be the primary mechanism of generation of freak waves.

The probability distribution of extreme wave heights suggested by Rayleigh, Ahn, and Mori can be used to predict the probability occurrence of freak waves. However, there are no definite measures available in comparing the accuracy in predicting the freak waves among three distribution function. For doing this we need more wave data needed to compare the performance of the predicting capability. Especially, long term wave data which satisfy the stationarity of time series is necessary.

The occurrence probabilities of extreme waves including two freak waves are represented as percentage in Table 5. It is found that the probability of occurrence of freak wave is much less than that of exceedence probability of $2 H_{1 / 3}$. This simply indicates that the frequency of freak waves is more rarer than those of $2 \mathrm{H}_{1 / 3}$. All 3 different distributions correctly discriminate the freak waves with the probability of occurrence of less than $3 \%, 0.1 \%$, and $8 \%$, respectively. We may utilize the probability density function of extreme waves to predict the occurrence probability of freak waves at specific location in the ocean.

\section{REFERENCES}

Ahn, K. 2002. Probability distribution of extreme wave heights in finite water depth, Proceedings of $29^{\text {th }}$ International Conference on Coastal Engineering, ASCE, 614-625.

Henderson, K.L, D.H. Peregrine and J.W. Dold. 1999. Unsteady water wave modulations: fully nonlinear solutions and comparison with the nonlinear Schrödinger equation. Wave Motion 29, 241-361.

Janssen, P.A.E.M. 2003. Nonlinear four-wave interactions and freak waves, Journal of Physical Oceanography 33, 863-884.

Kharif, C. and E. Pelinovsky 2003. Physical mechanisms of the rogue wave phenomenon. European Journal of Mechanics - B/Fluids 22 (6), 603-634. 
Liu, P.C., H.S. Chen, H.J. Doong, C.C. Kao and Y.J. Hsu 2009. Freque waves during Typhoon Krosa, Annales Geophysicae, 27, 2633-2642.

Mori, N. 2004. Occurrence probability of a freak wave in a nonlinear wave field, Ocean Engineering, $31,165-175$.

Mori, N. and P.A.E.M Janssen 2006. On kurtosis and occurrence probability of freak waves. Journal of Physical Oceanography 36, 1471-1483

Mori, N., P. Liu, and Y. Yasuda 2002. Analysis of freak wave measurements in the Sea of Japan, Ocean Engineering, 29 (11), 1399-1414.

Olagnon, M. and Athanassoulis, G. (Eds.) 2000. Rogue waves. IFRMER, France.

Ochi, M. 1998. Ocean waves: The stochastic approach, Cambridge University Press

Stansell, P. 2005. Distributions of extreme wave crest and trough heights measured in the North Sea. Ocean Engineering 32, 1015-1036.

Yasuda, T. and N. Mori 1997. Occurrence properties of giant freak waves in sea area around Japan. Journal of Waterways, Port, Coastal and Ocean Engineering 123 (4), 209-213. 